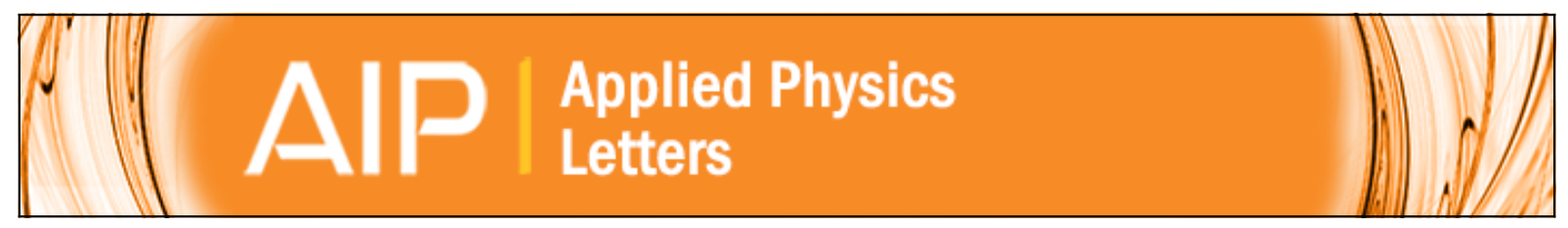

\title{
Electron transport in AIGalnP quantum well lasers
}

S. A. Wood, C. H. Molloy, P. M. Smowton, P. Blood, D. J. Somerford, and C. C. Button

Citation: Applied Physics Letters 75, 1748 (1999); doi: 10.1063/1.124807

View online: http://dx.doi.org/10.1063/1.124807

View Table of Contents: http://scitation.aip.org/content/aip/journal/apl/75/12?ver=pdfcov

Published by the AIP Publishing

\section{AlP Re-register for Table of Content Alerts}

\section{Create a profile. \\ Sign up today!}




\title{
Electron transport in AIGalnP quantum well lasers
}

\author{
S. A. Wood, C. H. Molloy, P. M. Smowton, P. Blood, ${ }^{\text {a) }}$ and D. J. Somerford \\ Department of Physics and Astronomy, Cardiff University, P.O. Box 913, Cardiff, CF2 3YB, \\ United Kingdom \\ C. C. Button \\ EPSRC Central Facility for III-V Semiconductors, University of Sheffield, Sheffield, S1 3JD, \\ United Kingdom
}

(Received 12 April 1999; accepted for publication 20 July 1999)

\begin{abstract}
By placing a direct-gap monitor layer (or collector) in the p-cladding layer of red-emitting AlGaInP laser diode structures, we have studied the transport of electrons through this layer by observation of spontaneous emission. Pulsed optical excitation superimposed on cw electrical injection has been used to determine the delay time between optical injection of carriers into the well and radiative recombination from the monitor pit. Computer simulations using measured values of minority carrier lifetime for the well and monitor layer show that the transit time for electrons through the p-cladding layer correspond to an electron mobility of $160 \mathrm{~cm}^{2} / \mathrm{V} \mathrm{s}$. (C) 1999 American Institute of Physics. [S0003-6951(99)02238-X]
\end{abstract}

Red-emitting AlGaInP laser diodes suffer from thermally activated leakage of electrons from the active region through the $p$-cladding layer which increases the threshold current and degrades the differential quantum efficiency. ${ }^{1,2}$ These effects are particularly pronounced in short wavelength devices (below about $640 \mathrm{~nm}$ ). The process is illustrated in Fig. 1, which shows the conduction band as a function of distance through the $p$-cladding layer and the active region of the device calculated by a self-consistent simulation $^{3}$ (Fig. 1 is discussed in greater detail below). It has been established that leakage of electrons occurs by diffusion and drift through the $X$-conduction band minimum, ${ }^{4,5}$ the importance of the drift contribution depending upon the $p$-doping density of the cladding layer. One aspect of this carrier leakage process which has not received attention is its impact on the modulation response of laser diodes. The response of the light output to modulation of the terminal voltage is determined by the ability of the carrier density in the well to respond to these voltage changes and one of the factors which controls this is the leakage of electrons through the cladding layer. Since this leakage occurs through the $X$-conduction band minima, where the carrier mobility is low, this process is slow. In principle it is possible to simulate this process, however the response time is dependent upon the value of the electron mobility in $p$-type AlGaInP which is not well known. This mobility controls the rate of diffusion and drift.

We have addressed this question directly by a "time-offlight" experiment in which we perturbed the carrier population in the quantum well of an electrically driven laser by means of a short optical pulse and observed the resulting optical response of a direct-gap monitor layer placed between the cladding layer and contact layer to detect the leaking electrons, as indicated in Fig. 1. We observe a time delay between the optical excitation and the response of the monitor layer and by means of a rate equation analysis of the

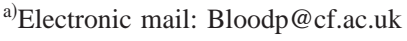

experiment we have been able to separate the effects of the carrier lifetimes (which determine the rise time of the response) from the time delay due to carrier transport.

The devices used in this investigation were typical visible-emitting laser structures, grown by metalorganic chemical vapor deposition with the monitor structure shown in Fig. 1 inserted between the $p$-cladding and contact layers. The lasers consist of an 80 - $\AA$-wide, compressively strained $\mathrm{Ga}_{0.38} \mathrm{In}_{0.62} \mathrm{P}$ quantum well set in a region of $\left(\mathrm{Al}_{0.15} \mathrm{Ga}_{0.85}\right)_{0.51} \mathrm{In}_{0.49} \mathrm{P}$, together forming the 2000- $\AA$-wide waveguide core, clad by $1-\mu \mathrm{m}$-thick layers of $\left(\mathrm{Al}_{0.7} \mathrm{Ga}_{0.3}\right)_{0.51} \mathrm{In}_{0.49} \mathrm{P}$ doped $9 \times 10^{17} \mathrm{~cm}^{-3} n$ type and 5 $\times 10^{17} \mathrm{~cm}^{-3} p$ type, respectively. The monitor structure consists of $5000 \AA$ of direct gap $\left(\mathrm{Al}_{0.3} \mathrm{Ga}_{0.7}\right)_{0.51} \mathrm{In}_{0.49} \mathrm{P}$ as a collector for electrons and barrier of $2000 \AA$ of $\left(\mathrm{Al}_{0.7} \mathrm{Ga}_{0.3}\right)_{0.51} \mathrm{In}_{0.49} \mathrm{P}$, both doped to $5 \times 10^{17} \mathrm{~cm}^{-3}$ using zinc. Figure 1 illustrates how electrons leaking through the $X$ minima of the $p$-cladding thermalize to the $\Gamma$ band in the monitor layer to recombine radiatively. The $\left(\mathrm{Al}_{0.3} \mathrm{Ga}_{0.7}\right)_{0.51} \mathrm{In}_{0.49} \mathrm{P}$ monitor layer is transparent to light from the rest of the structure and spontaneous emission from it can be spectrally resolved from that occurring elsewhere in

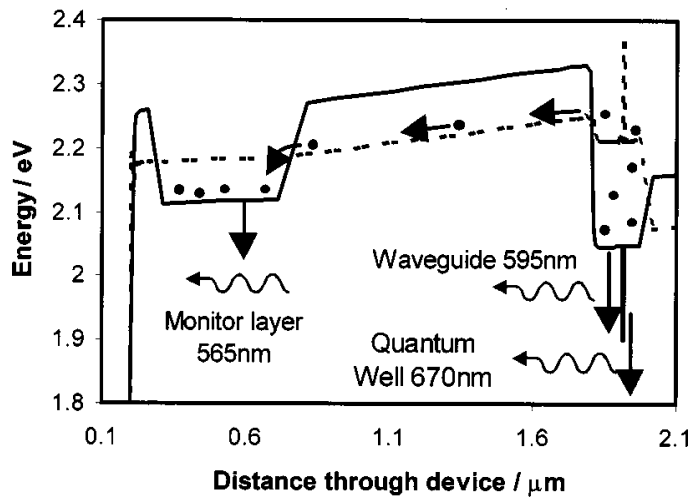

FIG. 1. Calculated conduction band cross section through the $p$ cladding and core region of a typical monitor pit laser at threshold. The solid line represents the direct band gap while the dotted line shows the indirect band gap. 
the device: the emission wavelengths for the quantum well, barrier, and monitor layer are given in Fig. 1. We have established that the introduction of the monitor layer does not affect the threshold characteristic of the device. From steadystate studies of these structures we have confirmed the presence of a drift component to the leakage current. ${ }^{4}$

Electron-hole pairs were generated in the quantum well by optical excitation through a window in the top $50-\mu \mathrm{m}-$ wide contact of a stripe geometry laser diode using $20 \mathrm{ps}$ pulses at a repetition rate of $4.7 \mathrm{MHz}$ and wavelength of 630 $\mathrm{nm}$ from a dye laser. This wavelength corresponds to excitation at photon energy just below the band gap of the barrier. Spontaneous emission from the optically active regions of the device was observed through the same contact window. ${ }^{6}$ With optical injection alone, no spontaneous emission was observed from the monitor region, which is as expected under low-injection conditions when the electron quasi-Fermi level is far below the $X$-band edge and there is no electric field across the cladding layer. When a forward bias was applied we observed a steady luminescence from the optically active components of the structure, including the monitor region, due to electrical injection and a superimposed transient response synchronous with the optical injection pulse. This paper is concerned with the transient emission from the monitor layer and in particular with the time of flight of electrons through the cladding layer.

For the temporal response experiments, the laser diode was driven electrically $\mathrm{cw}$ in forward bias with pulsed optical excitation as described above. Spontaneous emission from the monitor layer was detected at the peak of its spectrum at $585 \mathrm{~nm}$ and the time dependence determined by a standard time-resolved photon-counting system triggered by the optical excitation pulse. Figure 2 shows the time dependence of the logarithm of the monitor layer emission at room temperature for electrical injection currents of 20 and $50 \mathrm{~mA}$ and the signal due to pump light scattered from the surface of the sample. This provides a time reference and an indication of the overall time response of the measurement system. These data were corrected for the wavelength dependence of the transit time of electrons through the photomultiplier tube, which was determined experimentally for this system. The monitor layer emission comprises a steady background luminescence signal due to the continuous electrical injection, with the transient response to the pulsed optical injection into the well superimposed on this. The traces in Fig. 2 are normalized to the same peak signal level and, because the background level at $50 \mathrm{~mA}$ is greater than at $20 \mathrm{~mA}$, the amplitude of the transient signal as a proportion of the total signal is reduced. The response of the monitor layer is delayed approximately $0.5 \mathrm{~ns}$ relative to the reference signal at both injection levels. Repeated measurements from other devices prepared from the same wafer gave identical results for the transient response.

The time delay between the optical injection of carriers into the well and the resulting luminescence from the monitor layer is due to some or all of three components: (i) the rise time of the carrier population in the well, determined by the pulse shape and the carrier lifetime in the well, (ii) the transit of electrons through the $1-\mu \mathrm{m}$-thick $p$-cladding layer, and (iii) the rise time of the carrier population in the monitor

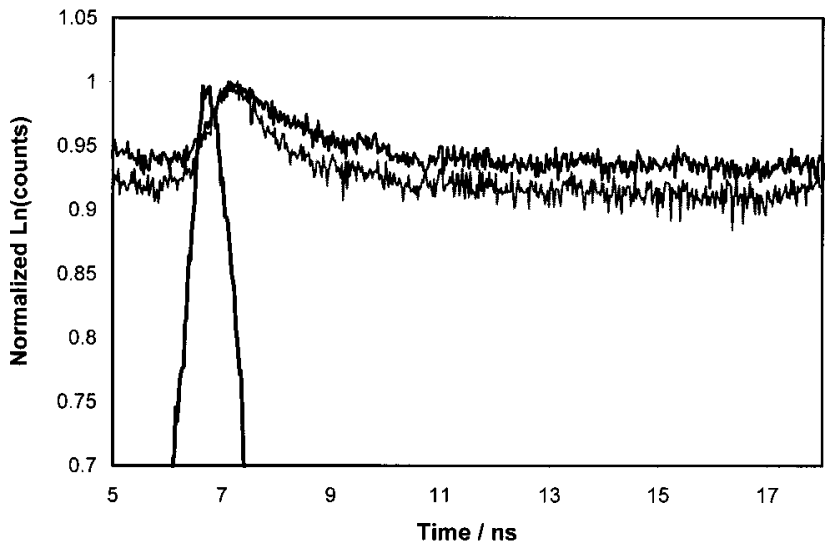

FIG. 2. Two time-of-flight measurements at room temperature showing the $0.5 \mathrm{~ns}$ separation recorded between the fluctuation produced in the monitor pit, by optical injection into the quantum well in conjunction with a $20 \mathrm{~mA}$ cw background, (lower trace) and at $50 \mathrm{~mA} \mathrm{cw}$ background, (upper trace), relative to the pump pulse (spike).

region. To interpret the data to determine the contributions of these processes we performed a computer simulation of the experiment, including the temporal response of the measurement system as indicated by the scattered pump signal. To provide reliable input data we measured the carrier lifetimes in the well and monitor layer in an unbiased device by a standard time-resolved luminescence experiment. The values obtained were short compared to the low-injection radiative lifetimes and were indicative of the nonradiative rates. The short value for the monitor layer $(0.5 \mathrm{~ns})$ was probably due to its relatively high $\mathrm{Al}$ content, while the nonradiative lifetime of the confined electrons in the well (1 ns) was determined in part by tunneling into the Al-containing barrier layers where recombination occurs. Based on these data we took a value of $0.25 \mathrm{~ns}$ for the nonradiative lifetime of electrons in the $X$ band of the cladding layer. We assumed that the times for thermalization of the carrier distribution in the well and for the capture and thermalization of electrons in the monitor layer are short (of order $10 \mathrm{ps)} \mathrm{compared} \mathrm{with}$ the carrier lifetimes and the transit time. Nominal values were used for the layer compositions and the $p$-doping density was set to $5 \times 10^{17} \mathrm{~cm}^{-3}$ as determined from capacitance-voltage profiling. The cladding layer thickness was $1 \mu \mathrm{m}$.

In the simulation, the carrier populations in the well and barrier/waveguide core had spatially uniform thermal energy distributions and were coupled through standard processes of capture and thermal emission. ${ }^{7}$ Following previous experiments, ${ }^{5}$ we regard the leakage as occurring through the $X$ conduction band minimum of the $p$-cladding layer. We therefore calculated the carrier population in the waveguide core at and above the energy of the $X$-band edge. This population was used in the first element of a finite element treatment of carrier flow through the cladding layer using the standard continuity equation with drift, diffusion, and recombination. ${ }^{8}$ Numerical values for the parameters used are given in Table I. Once the model reached a steady state at the specified current, a Gaussian temporal pulse of minority carriers was added to the quantum well and the time evolution of the carrier populations through the structure was computed. Figure 3 shows the simulated scattered pump light, 
TABLE I. Material input parameters used in the monitor layer device simulations.

\begin{tabular}{llll}
\hline \hline Symbol & \multicolumn{1}{c}{ Definition } & \multicolumn{1}{c}{ Value } & \multicolumn{1}{c}{ Units } \\
\hline$\mu_{e}$ & Minority carrier mobility & 160 & $\mathrm{~cm}^{2} / \mathrm{V} \mathrm{s}$ \\
$\mu_{p}$ & Hole mobility & 10 & $\mathrm{~cm}^{2} / \mathrm{V} \mathrm{s}$ \\
$\tau_{\text {nrqw }}$ & Nonradiative lifetime in quantum well & $1 E-9$ & $\mathrm{~s}$ \\
$\tau_{\text {nrwg }}$ & Nonradiative lifetime in waveguide & $0.5 E-9$ & $\mathrm{~s}$ \\
$\tau_{\text {nrclad }}$ & Nonradiative lifetime in $p$ cladding & $0.25 E-9$ & $\mathrm{~s}$ \\
$\tau_{\text {cap }}$ & Capture lifetime into $\Gamma$ band of monitor layer & $1 E-12$ & $\mathrm{~s}$ \\
$\tau_{\text {rmp }}$ & Radiative lifetime in monitor layer & $1 E-9$ & $\mathrm{~s}$ \\
$\tau_{\text {nrmp }}$ & Nonradiative lifetime in monitor layer & $1 E-9$ & $\mathrm{~s}$ \\
$\alpha_{m}$ & Mirror loss & 26.75 & $\mathrm{~cm}^{-1}$ \\
$\alpha_{i}$ & Internal scattering loss & 10 & $\mathrm{~cm}^{-1}$ \\
\hline \hline
\end{tabular}

and the luminescent response of the monitor layer with some parameters (primarily carrier mobilities) adjusted to match the experimental data. The experimental data with the background level removed are also plotted (thin line), illustrating the excellent agreement obtained between simulated and experimental data. The rise time of the simulated carrier population in the well is of order $20 \mathrm{ps}$, determined by the width of the exciting pulse since this is much shorter than the carrier lifetime. The rise time at the monitor layer is determined by the transport processes whereas the decay time is determined primarily by the decay time of the driving carrier population in the well because the well lifetime is longer than that in the monitor layer. We found that delay time was independent of the monitor layer nonradiative lifetime for values above $0.2 \mathrm{~ns}$. The transit of electrons through the cladding layer makes the largest contribution to the delay.

We investigated the sensitivity of the delay time to the values used for the hole and electron mobilities in the $p$-cladding layer. Values for the hole mobility in the cladding layer material are not well known. The hole mobility in the cladding layer controls the field at a given drive current, and

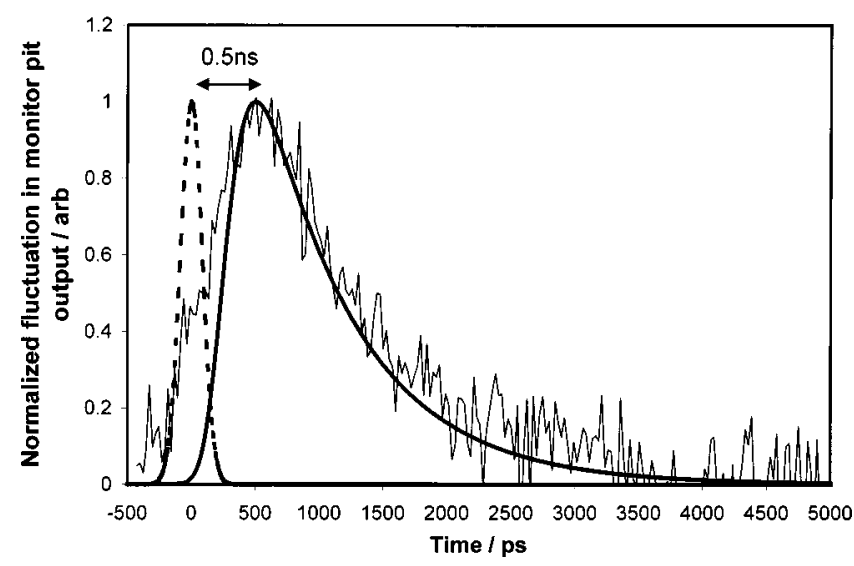

FIG. 3. Simulated time-of-flight data produced at $300 \mathrm{~K}$. A $20 \mathrm{~mA}$ background $\mathrm{cw}$ injection was used in conjunction with a hole mobility of 10 $\mathrm{cm}^{2} / \mathrm{V} \mathrm{s}$ and an electron mobility of $160 \mathrm{~cm}^{2} / \mathrm{V}$ s. The nonradiative lifetime in the monitor pit was set to $1 \mathrm{~ns}$. A peak to peak separation of $0.5 \mathrm{~ns}$ was recorded. The $20 \mathrm{~mA}$ experimental data are included (thin line), with the background level removed for comparison. was varied from 5 to $25 \mathrm{~cm}^{2} / \mathrm{V} \mathrm{s} .^{9}$ Only for a mobility below $10 \mathrm{~cm}^{2} / \mathrm{V} \mathrm{s}$ did we find an effect, with the delay time becoming shorter as the mobility was reduced due to the increase in electric field as the hole conductivity is decreased. The hole mobility influences electron drift rate but not electron diffusion. The value was fixed at $10 \mathrm{~cm}^{2} / \mathrm{V} \mathrm{s}$ for the remainder of the simulations. The electron mobility influences both drift and diffusion and was varied from 60 to $180 \mathrm{~cm}^{2} / \mathrm{V} \mathrm{s}$. The delay was strongly dependent on the electron mobility with the delay time decreasing as the electron mobility was increased. The time of flight was found to change by $\sim 0.4 \mathrm{~ns}$ over the range of electron mobility values tested. We find that an electron mobility of $160 \mathrm{~cm}^{2} / \mathrm{V} \mathrm{s}$ gave the measured delay of $0.5 \mathrm{~ns}$ and therefore we conclude that this is the value of the electron mobility in this material. This value is slightly higher yet comparable with previously published values of $100 \mathrm{~cm}^{2} / \mathrm{V} \mathrm{s}$ for the minority carrier mobility in similar $p$-cladding compositions. ${ }^{1}$ The simulation does not predict any measurable change in the delay time for the two currents of 20 and $50 \mathrm{~mA}$ because the transport occurs by a mixture of drift and diffusion.

Using a direct-gap monitor layer (or collector), minoritycarrier transport through the $p$-cladding layer of AlGaInP laser diodes has been investigated by observation of spontaneous emission through a top-contact window. A combination of pulsed optical excitation superimposed on cw electrical injection has been used to determine the delay time between optical injection of carriers into the quantum well and radiative recombination from the monitor pit. The average delay time was measured to be $0.5 \mathrm{~ns}$ for drive currents of 20 and $50 \mathrm{~mA}$. By means of a computer simulation using measured values of minority carrier lifetime for the well and monitor layer, we find that the transit time for electrons through the $p$-cladding layer is the major contributor to the observed delay time and that the simulated delay is strongly dependent upon the electron mobility. An electron mobility of $160 \mathrm{~cm}^{2} / \mathrm{V} \mathrm{s}$ was required to match the time of flight measured experimentally.

We thank P. J. Hulyer for his skilful fabrication of the devices used in these experiments. We thank the Engineering and Physical Sciences Research Council for their support of this project and for a studentship for SAW.

${ }^{1}$ D. P. Bour, D. W. Treat, R. L. Thornton, R. S. Geels, and D. F. Welch, IEEE J. Quantum Electron. 29, 1337 (1993).

${ }^{2}$ P. M. Smowton and P. Blood, IEEE J. Sel. Top. Quantum Electron. 3, 491 (1997).

${ }^{3}$ D. L. Foulger, P. M. Smowton, P. Blood, and P. A. Mawby, IEE Proc.: Optoelectron. 144, 23 (1997).

${ }^{4}$ S. A. Wood, P. M. Smowton, C. H. Molloy, P. Blood, D. J. Somerford, and C. C. Button, Appl. Phys. Lett. 74, 2540 (1999).

${ }^{5}$ P. M. Smowton and P. Blood, IEEE J. Quantum Electron. 31, 2159 (1995).

${ }^{6}$ P. Blood, A. I. Kucharska, J. P. Jacobs, and K. Griffiths, J. Appl. Phys. 70, 1144 (1991)

${ }^{7}$ H. Schneider and K. V. Klitzing, Phys. Rev. B 38, 6160 (1988).

${ }^{8}$ D. Wood (Prentice Hall International, 1994).

${ }^{9}$ Y. Ohba, M. Ishikawa, H. Sugawara, M. Yamamoto, and T. Nakanisi, J. Chem. Phys. 77, 374 (1986). 